Cinémas

Revue d'études cinématographiques

Journal of Film Studies

\title{
Le paysage dans la tradition documentaire québécoise : un regard off sur la parole
}

\section{Michèle Garneau}

Volume 12, numéro 1, automne 2001

Le Paysage au cinéma

URI : https://id.erudit.org/iderudit/024871ar

DOI : https://doi.org/10.7202/024871ar

Aller au sommaire du numéro

Éditeur(s)

Cinémas

ISSN

1181-6945 (imprimé)

1705-6500 (numérique)

Découvrir la revue

Citer cet article

Garneau, M. (2001). Le paysage dans la tradition documentaire québécoise : un regard off sur la parole. Cinémas, 12(1), 127-143.

https://doi.org/10.7202/024871ar
Résumé de l'article

On dégagera ici trois esthétiques du paysage à l'intérieur d'une même tradition cinématographique, celle du documentaire québécois en style " direct ».

L'hypothèse est que le lieu du commentaire - de la traditionnelle voix off - s'est déplacé du côté du regard et qu'il doit être débusqué dans un paysage. Ce paysage comme visibilité de la parole, nous l'examinerons d'abord chez Pierre Perrault, pour ensuite nous pencher sur deux héritiers contemporains de cette tradition : Lucie Lambert et Sylvain L'Espérance. 


\title{
Le paysage dans la tradition documentaire québécoise: un regard off sur la parole
}

\section{Michèle Garneau}

\begin{abstract}
RÉSUMÉ
On dégagera ici trois esthétiques du paysage à l'intérieur d'une même tradition cinématographique, celle du documentaire québécois en style "direct». L'hypothèse est que le lieu du commentaire - de la traditionnelle voix off - s'est déplacé du côté du regard et qu’il doit être débusqué dans un paysage. Ce paysage comme visibilité de la parole, nous l'examinerons d'abord chez Pierre Perrault, pour ensuite nous pencher sur deux héritiers contemporains de cette tradition: Lucie Lambert et Sylvain L'Espérance.
\end{abstract}

\section{ABSTRACT}

This article takes up a discussion of three different landscape aesthetics within the same film tradition, that of the Québécois "cinéma direct" documentary. The author's hypothesis is that the space of the commentary, of the traditional voice over, has been displaced here to the realm of the gaze, and that it can be uncovered in the landscape. This landscape, a speech made visible, is examined first of all in the work of Pierre Perrault. Discussion then focuses on two contemporary heirs to this tradition, Lucie Lambert and Sylvain L'Espérance.

\section{Postulat collectiviste et puissance d'oralité}

Une des tendances les plus profondes, parce que inaugurale, du documentaire québécois repose sur un postulat "collectiviste", à 
savoir: n'importe qui est capable de raconter. C'est par la pratique d'un cinéma direct, dont on sait qu'il aura permis, trente ans après le cinéma parlant, un cinéma de la "prise de parole" "que la cinématographie québécoise se dote d'une première identité poétique ${ }^{2}$.

Ce que libéreront les innovations techniques du cinéma direct, c'est une puissance d'oralité. À l'Office national du film du Canada (ONF), foyer de la tradition documentaire canadienne, le «16-direct québécois» bouleverse les conventions du genre. Ce qui est touché principalement, voire considérablement miné par cette nouvelle puissance cinématographique, c'est le pouvoir de la voix off. On peut même considérer sa destitution comme le trait le plus remarquable des premiers documentaires québécois. La voix off est destituée au profit d'une parole populaire qui constitue désormais le tout — et la finalité - de l'énonciation documentaire. La révolution est carnavalesque: des actes de parole, issus du bas, viennent détrôner la Voix venue d'en haut, et établir le règne d'une véritable énonciation collective. En d'autres termes: Dieu n'est plus l'auteur des documentaires ${ }^{3}$, mais le peuple. C'est lui, désormais, qui (se) raconte.

Une tradition dans la manière de filmer ${ }^{4}$ s'implante, dans laquelle ce qui structure l'énonciation documentaire, c'est un montage de paroles suscitées par le filmage lui-même, et non plus une voix off surajoutée et montée sur des images. Or, cette nouvelle pratique cinématographique, où l'image n'est plus commentée, a-t-elle pour autant invalidé le commentaire, cet espace du commentaire que représente la voix off? Mon hypothèse est que non, et l'on me suivra dans ma démonstration si l'on veut bien admettre qu'un commentaire, au cinéma, n'est pas nécessairement verbal, c'est-à-dire porté par une voix, mais qu'il peut être visuel, c'est-à-dire porté par un regard. L'image s'étant remplie de voix, l'enjeu n'est plus de la faire parler par un commentaire verbal, mais de faire voir la parole par un commentaire visuel. C'est le regard qui doit désormais réfléchir les voix et non plus la voix réfléchir (sur) l'image. Car c'est bien dans la réflexivité que se loge le pouvoir de la voix off, pouvoir d'instituer un lieu de distance réflexive par rapport à ce qui s'inscrit dans la bande-image. La possibilité de cette distance réflexive n'a donc pas disparu de la nouvelle pratique du documentaire, qui a mis la voix off au 
rancart: elle est seulement passée du côté du regard. C'est une distance instituée par un regard sur ce que l'on entend et non plus une distance instituée par une voix sur ce que l'on regarde.

Ce qui a changé, fondamentalement, dans le passage d'un commentaire verbal, porté par la voix, à un commentaire visuel, porté par le regard, c'est le régime d'explication auquel ce nouveau type de commentaire nous convie. "Expliquer veut dire deux choses bien différentes, écrit Jacques Rancière. Ce peut être donner le sens d'une scène, la raison d'une attitude ou d'une expression. Mais ce peut être, selon l'étymologie du mot, lui laisser dérouler la plénitude enroulée dans sa simple présence» (1997, p. 53). Le pouvoir explicatif que la voix off s'octroie sur le déroulement de l'image, correspond au premier régime du mot: apposer un sens... donner la raison de... Il s'agit de la fonction la plus courante du documentaire, à savoir sa fonction cognitive, fonction qui passe, le plus souvent, par la voix off narrative ${ }^{5}$. Haut lieu du sens dans le documentaire traditionnel, la voix off est aussi et surtout un lieu autre du sens. C'est le lieu du verbe dont on connaît la supériorité réflexive sur l'image, sa hauteur de vue sur "les vues".

Avec le deuxième régime du mot, et qui correspond, en fait, à sa définition originaire, "expliquer" prend un sens plus artisanal qu'analytique: il s'agit de "déplier", de "dérouler ${ }^{6}$ ». Si le paysage "explique" ici la parole, c'est en tant qu'il lui "laisse dérouler la plénitude enroulée dans sa simple présence». Le lieu du commentaire n'est plus rationnel, porté par le verbe (logos), mais sensible, porté par une image-paysage (pathos). Il s'agit donc d'un déplacement qui fait passer le sens (la parole) dans le sensible (un paysage).

Pierre Perrault filme la parole comme une véritable matière en mouvement. Chez lui, les voix ne cessent de se détacher des corps pour aller se raccrocher à un paysage. Ce mouvement de déterritorialisation de la parole (de son lieu d'origne) et de reterritorialisation (dans un lieu autre de distance réflexive qu'est le paysage), on le retrouve aussi chez Lucie Lambert et Sylvain L'Espérance. C'est donc essentiellement en la réfléchissant dans un lieu autre, c'est-à-dire en l'exilant de son lieu d'origine (visage), que la parole peut être «expliquée». «La déterritorialisation, 
nous disent Deleuze et Guattari, est toujours double... Elle comporte toujours une force déterritorialisante [en l'occurrence ici, le paysage (M. G.)] et une force déterritorialisée [en l'occurrence ici, une parole (M. G.)]" (1980, p. 377).

Chez Perrault, ce que le paysage doit réfléchir, ce sont les voix de ceux qui l'habitent. Mais là s'arrête, pour ainsi dire, sa réflexivité. Chez Lucie Lambert et Sylvain L'Espérance, en revanche, une double réflexivité est à l'œuvre. S'il vient, comme chez Perrault, réfléchir ce qu'expriment les personnages, le paysage fonctionne aussi comme lieu de réflexion plus personnelle de l'auteur. C'est là où la pratique du paysage de ces deux cinéastes differe de celle de Perrault, dans la mesure où le paysage devient le lieu d'une intervention du cinéaste, et exprime une émotion ou une pensée.

\section{Médium et pouvoir médiumnique}

Aucun paysage, chez Perrault (1995, p. 21), ne saurait rester muet :

J'ai reconnu ce grand vide de mon propre paysage. L'ayant parcouru dans sa géographie. Ne l'ayant pas rencontré dans les écritures. Pays sans parole? Ou plutôt sans écriture. Mais il restait la parole justement qui m’a permis de résoudre mon absence. La belle chouenne populaire. J'avais donc à explorer l'immense territoire de l'oralité.

Ce "grand vide» d'un paysage que le cinéaste-poète porte en lui comme une absence - il faudrait dire, qui l'absente à luimême - seule la parole populaire pourra venir le combler. Le paysage ne devient pays, c'est-à-dire habité, qu'en sortant de son mutisme, qu'en se remplissant des voix de ceux qui l'habitent. C'est pourquoi il faut aller vers l'habitant, vers celui qui s'est familiarisé avec un paysage et qui, dès lors, peut le nommer. "J'étais un étranger dans un paysage encore innommé", écrit Perrault (1985, p. 145). Vide, absence, étrangeté, voilà ce qu'inspire d'abord le paysage au cinéaste. Pierre Perrault a horreur du vide. Partout il cherche les traces d'une appropriation physique et méta-physique du territoire, ce qu'il appelle "l'occupation fondamentale». Le réel chez Perrault, c'est la trace. Dans 
les plis d'un territoire, et comme tapies dans le matériau, il y a les traces d'une fréquentation de longue date, de tous ceux qui ont hanté (au sens de "fréquenter") un paysage. Et ce dont témoignera le film, ce n'est pas seulement, à travers la trace, de l'expérience humaine d'un lieu, mais aussi d'une hantise des lieux (cette fois-ci au sens anglais de "haunted") par le cinématographe. Que la caméra soit ce médium au pouvoir médiumnique, c'est-à-dire d'un pouvoir de communiquer, à travers la trace physique, avec un esprit des lieux, c'est ce que démontrent tous les itinéraires - terriens ou marins - de Pierre Perrault. Toute l'œuvre est une hantise du fleuve, de la forêt et de la terre.

Il y a d'abord les traces de l'origine, de tous ceux qui ont ouvert le pays, tous ces découvreurs et défricheurs. Les projets de Les Voiles bas et en travers (1983) et de La Grande Allure (1985), cycle sur le fleuve Saint-Laurent, sont de refaire, sur les traces de Jacques Cartier, le grand voyage des "découvrances", selon l'expression de Perrault. "Je cherche un fleuve symbolique ", déclare le poète du cycle, un fleuve que la caméra suscitera à partir de diverses traces glanées ici et là au cours du voyage. Dans chaque cycle, Perrault se "territorialise", il entre dans un agencement territorial. Et ce qui doit en sortir, ce qui doit être dégagé de ce coin de pays que le cinéaste a élu, c'est une mémoire, toute une puissance spirituelle. "Il faudrait qu'il y ait des dieux dans le Saint-Laurent", avance Michel Serres dans $L a$ Grande Allure. On ne saurait mieux résumer la quête. Car tout l'effort cinématographique est de faire lever, de soulever de terre ou de mer - et à travers la trace physique - une puissance méta-physique, un esprit des lieux.

"On a voulu suivre la piste du caribou et des mémoires", écrit Perrault au sujet du Pays de la terre sans arbre. Une double piste, mais dont la première est indispensable au surgissement de la seconde. Commentant son film Un pays sans bon sens (1970), Perrault écrivait: "Cette humble tentative de pays difficile à dire... et les caribous à notre rescousse pour exprimer l'inaliénable» (1972, p. 29). Dans le cycle montagnais, le temps lourd, le temps quasi immobile de la toundra figée dans son archaïsme se mesure ici au quotidien des Montagnais, à celui de la réserve. Il est la surface d'inscription du temps, et surtout de la mémoire 
fragile. Ce territoire, il y a très longtemps, a été aimé, exploité, habité, voire symbolisé. Des dieux l'habitent encore, mais il sont en train de disparaître à jamais. Pour que le récit survienne, celui de la légende d'Atticknapéo, le dieu du caribou, il faut aller sur ces lieux de mémoire, lieux qui en ont permis l'invention, dans ce paysage de la toundra, aux abords du lac Mouchouânipi, et la possibilité de recréer, par la caméra, tous les gestes qui en permettront le surgissement. La trajectoire de la caméra dans le paysage tente d'illustrer l'évocation mentale. C'est une image mentale, mais que seul un paysage peut porter. Il y a ici tout un maillage de parcours dans une immense découpe de l'espace et du temps qui fait lire comme une carte. "Le Pays de la terre sans arbre est un chef-d'œuvre, écrit Deleuze, où se juxtaposent les images géographiques, cartographiques, archéologiques, sur le parcours devenu abstrait du caribou" (1985, p. 318).

Pays et paysage, chez Pierre Perrault, sont une seule et même chose. Mais cette remontée étymologique (de paysage à pays) n'est possible que par un retour aux sources de l'oralité. Le cinéma de Pierre Perrault, c'est la quête d'un pays(age) que tout l'effort cinématographique consistera à élever au statut de parole. Ce cinéma de la parole n'est pas seulement celui, comme on l'a répété, de la parole des gens. Il s'agit aussi de faire parler ce qui est muet. Perrault trouve de la parole partout. Toute réalité matérielle, en l'occurrence le paysage, est élevée au statut de parole. Les derniers films testamentaires, véritable géologie de l'esprit, en font foi.

Il y a les paysages encore habités: alors on parle dans un paysage, on en fait organiquement partie. C'est le premier cycle, celui de l'île aux Coudres, où toutes les conditions d'une organicité communautaire étaient réunies. Il y a ceux qui ne le sont plus, ou presque plus. Ce sont les paysages qui se vident ou qui se sont vidés de leurs occupants. On parle alors moins dans les paysages que pour eux, on les poursuit. Un désœuvrement gagne la suite de l'œuvre. Dans les paysages que va élire le cinéaste après Un pays sans bon sens — la terre abitibienne des paysans délocalisés, la toundra désertée des Montagnais -, la puissance d'expression ne se dégage plus aussi aisément du paysage, car pays, gens du pays, parole populaire et paysage ne forment plus un tout (totalité organique) harmonieux. Il y a comme une résistance des 
voix à s'élever de la terre. Et si les lieux gagnent en désertification, le langage, lui, gagne en aphasie. Son déroulement est plus ardu, et sa présence - celle d'une parole lumineuse en accord avec son paysage — n'est plus aussi pleine. La présence à soi dans un paysage est ici ce qui fait défaut. Car c'est la patrie qui, en définitive, est recherchée. La patrie, c'est le pays du père. C'est le patriote et le patrimoine. Ce n'est plus le lieu, mais le lien. À partir d'un lieu, c'est un lien que cherche à tisser Perrault, un sentiment d'appartenance à un lieu.

Partir en quête de la nomination du (pays)age à travers la parole vivante: telle sera la motivation initiale. Toute l'œuvre de Pierre Perrault est une pensée du/sur le paysage. Qu'il s'agisse de sa poésie, où il s'exprime en son propre nom, ou de son cinéma, où il se raconte au pluriel — "Je me raconte au pluriel, écrit Perrault. L'individu Perrault ne tient pas beaucoup de place. Et il ne le regrette pas. " (1983, p. 56) - ce qu'il s'agit d'exprimer, c'est un (pays)age.

Le cinéaste a-t-il résolu son absence, comblé le vide? Comment comprendre le retour de la voix off dans ses deux derniers documentaires? En effet, dans L'Oumigmatique ou l'objectif documentaire (1993) et Cornouailles (1994), la voix off a envahi le paysage, pour la première fois dans le cinéma de Pierre Perrault. Comment comprendre cette confrontation au désert, dans les derniers films testamentaires? Justement, en y voyant un testament, un art poétique où poésie et cinéma (celui qui permet une oralisation de la poésie) sont réunis. Dans ces films, c'est son émotion personnelle devant le paysage que nous livre Perrault. Le cinéaste part en quête d'un corps brut - le bœuf musqué - et expérimente, à partir de l'irréductibilité totale de l'animal préhistorique, des postures de cinéaste. Attente, guet, qui-vive, distance respectueuse, apparition, irruption, dispersion, nomination sont les éléments de cet art poétique que sont L'Oumigmatique ou l'objectif documentaire et Cornouailles. L'ouvre de Perrault est encadrée par deux corps purs: le corps commun communautaire de l'île aux Coudres - qu'il mettra en légende - et le corps sauvage du bœuf musqué - dont il attestera la mise en légende depuis le début de l'humanité. 


\section{Paysage et image-affection}

L'image ultime, l'image à constituer, dans tous les films de Pierre Perrault, est celle d'un pays(age). Dans les paysages de Lucie Lambert, si le pays a encore une place - l'attachement, dans Avant le jour, des gens à leur île - , ce n'est plus lui, cependant, qui motive la représentation. Ce qui sous-tend la poétique du paysage de Lucie Lambert, ce n'est plus un pays, mais une âme, un paysage intérieur, que suggère le très beau titre de son premier documentaire: Paysage sous les paupières. La formule bien connue d'Amiel, "le paysage est un état de l'âme", sied parfaitement aux deux films de la cinéaste. Ou encore, ce vers de Verlaine, tiré du célèbre Clair de lune: "Votre âme est un paysage choisi." "

Quel est ce paysage sous les paupières? Un passé lourd, une expérience familiale douloureuse, mais aussi, le désir de vivre, d'être enfin heureux. Des femmes se confient à la caméra, et lorsque la confession se fait trop déchirante, la caméra se retire pudiquement du visage, pour aller vers un paysage. La confession se poursuit, en voix off, mais elle se "déroule" désormais dans un paysage. Lucie Lambert mêle sa propre subjectivité, par ses images de paysage, à celle des gens qui se livrent à la caméra. Dans un moment d'émotion extrême face aux confidences d'une adolescente, le paysage, filmé derrière une vitre, perdra peu à peu ses contours pour s'irréaliser dans la blancheur. La cinéaste va en effet faire un zoom avant jusqu'à atteindre un point d'aveuglement, opérant ainsi ce que Gilles Deleuze appelle une "cristallisation". Ce regard-paysage sur la parole opère une temporalisation de l'image, venant ainsi conférer comme une sorte de poids d'être aux paroles proférées. Car la douleur est dans le cadre. Il s'agit d'une émotion puisée, si l'on peut dire, dans la substance même du regard. Dans de telles échappées pudiques, c'est sa visualité qui s'approfondit, sa matérialité. Cette crispation formelle du paysage communie, en quelque sorte, avec la douleur exprimée par les mots. "Et pourquoi l'expression n'arriverait-elle pas aux choses? écrit Deleuze. Il y a des affects de choses" (1985, p. 138).

Lucie Lambert construit une "image-affection", ce qui signifie qu'elle sépare l'affect de ce qui l'exprime. Dans l'imageaffection, nous dit Deleuze, il y a un affect (l'exprimé) et il y a 
ce qui l'exprime: un visage ou un paysage. Ce qui exprime un affect n'est donc n'est pas nécessairement lié à un visage. Ce peut être un équivalent, un "objet visagéifié " qui, comme un paysage, peut se tailler un espace. "L'affect, écrit Deleuze, est exprimé mais il n'existe pas indépendamment de quelque chose qui l'exprime, bien qu'il s'en distingue tout à fait " (1985, p. 138). C'est par là que le dédoublement du regard est possible, et que le paysage peut venir "expliquer" la parole recueillie, c'est-à-dire la méditer visuellement. Les îles enchanteresses de Lucie Lambert, dans Avant le jour, à la fois nous regardent et nous posent comme sujet du regard. "Cette chose, écrit Deleuze [en l'occurrence ici, un paysage, M. G.], a été traitée comme un visage, elle a été envisagée, ou plutôt visagéifiée, et à son tour elle nous dévisage, elle nous regarde... même si elle ne ressemble pas à un visage" (1985, p. 126).

Dans Paysage sous les paupières, comme dans Avant le jour, le paysage est souvent à lire comme lieu d'inscription d'affects: consolation, apaisement, baume, palliatif à la douleur. C'est le paysage comme refuge (comme chez les romantiques), la source d'une consolation et d'une purification. Les paysages-pathos de Lucie Lambert ont pour fonction de spiritualiser la nature, prétexte à l'expression d'un sentiment humain, parabole d'une joie, séjour d'un état de l'âme.

Il n'y a pas ici, comme chez Perrault, de mise en situation en vue de susciter la parole, ni non plus d'échanges dialogués. Des femmes se confient, souvent seules devant la caméra. Elles posent un regard sur elles-mêmes et sur le monde et, par les images de paysage, la caméra prolonge ce regard, lui donne de l'ampleur. Et bien que nous soyons encore, comme chez Perrault, dans "les travaux et les jours", - Avant le jour me semble presque un hommage au Règne du jour de Perrault -, l'univers est féminin, et non plus masculin. Commentant le cinéma de Perrault, Gilles Thérien écrit très justement: "C'était du cinéma pour "homme" avec une sorte de satisfaction virile à faire le film en même temps que le pays" (1999, p. 163-164). Dans l'approche plus féminine de Lucie Lambert, le silence occupe autant d'espace que la parole. Nous ne sommes plus conviés à une performance orale qui, chez Perrault, venait remplir l'image 
de paysage, mais à une sorte de stase du paysage, presque une extase. Mais s'il y a beaucoup de silence chez Lucie Lambert, il y a aussi beaucoup de musique. Ce n'est plus le même agencement "visage, paysage et parole" que chez Perrault. Le paysage est ici formé d'alliances surprenantes, car «chaque trait libéré de visagéité fait rhizome avec un trait libéré de paysagéité, de picturalité, de musicalité" (Deleuze, 1980, p. 232). Il faut voir comment Avant le jour libère des traits de visagéité comme des oiseaux.

L'image sonore s'est donc complexifiée, et le mixage est aussi important, à l'intérieur de ces films, que le montage, étant dit que le mixage ne comporte pas seulement une distribution des différents éléments sonores, mais l'assignation de leurs rapports, souvent différentiels, avec les éléments visuels. Perrault découpe et fragmente des échanges dialogués, mais c'est là l'essentiel de ce qui compose son image sonore. Tout se passe, chez Lucie Lambert, comme si le continuum sonore ne cessait de se différencier en empruntant plusieurs directions, l'une emportant des actes de paroles réflexifs, une autre de la musique, une autre des secrets ou des ritournelles d'enfants, une autre les bruits de la mer: vagues, cris des mouettes, etc.

La dimension affective a remplacé la dimension épique. Ce n'est plus le paysage comme visage de la patrie. Il s'agit bien plutôt de mouvements d'attraction entre un visage et un paysage, entre un paysage et un visage: "Quel visage n’a pas appelé les paysages qu'il amalgamait, la mer et la montagne, quel paysage n'a pas évoqué le visage qui l'aurait complété, qui lui aurait fourni le complément inattendu de ses lignes et de ses traits?» (Deleuze, 1980, p. 212).

Une vision cosmique du paysage s'oppose, par sa postulation vers le haut, à la vision géologique vers laquelle tendra Perrault de plus en plus. Les paysages de Lambert ont la fragilité d'un reflet dans l'eau. Une brume s'étale sur la grève et la cinéaste compose des marines. Puis, la caméra s'élève et filme le reflet, dans l'eau, du clair de lune. Nous ne sommes pas rendus à la terre, comme chez Perrault, mais à la mer, vers une calme puissance fluviale et maritime. Ajoutons: à la mère aussi. Dans Avant le jour, la première image est celle d'un écran noir et une 
femme raconte, émue, son accouchement. Où sommes-nous donc, dans cet "avant le jour", dans quel paysage, dans quelle obscurité? C'est à la vie, et non plus au pays, que la puissance féminine du paysage nous fait remonter.

\section{Paysage et exaspération de la technique}

Dans Les Printemps incertains, une femme chante une ritournelle gaélique devant le fleuve Saint-Laurent, à la mémoire des siens. La voix humaine, c'est ce qui s'oppose, dans Les Printemps incertains, aux coups de pioches des démolisseurs. Telle une âme errante, la ritournelle irlandaise hante les maisons détruites. Car il y a eu une vie ici, une vie de quartier. Le cinéaste filme les traces, tente de retracer ces lieux de vie, de les évoquer. Mais quand c'est la trace elle-même qui est enfouie, il n'y a plus que de la mémoire vivante, une mémoire dont des hommes sont encore porteurs.

Cette femme, filmée de dos, et qui regarde le fleuve SaintLaurent à la brunante, à quoi pense-t-elle? Ils sont sans doute arrivés par là, les siens, chassés par la grande famine. On les a mis en quarantaine et un grand nombre d'entre eux sont morts du typhus. On les a enterrés ici et là, comme on a pu. Quelqu'un raconte qu'en construisant le pont Victoria, on a trouvé des ossements. "Ils étaient six mille. Ils doivent être partout dans les parages.» Cette mémoire irlandaise du fleuve, ce n'est pas celle que recherchait Perrault. Les immigrants, la ville, l'usine, cet autre hors-champ du cinéma de Pierre Perrault, Sylvain L'Espérance l'assume. S'il n'y a pas de dieux dans le fleuve, il n'y en a pas moins une "mémoire des lieux», titre de la première partie des Printemps incertains. Nous resterons sur terre, près des cadavres enfouis sous la structure métallique du pont.

Nous tournons autour du canal Lachine, du Village-aux-Oies et de Pointe-Saint-Charles. Nous côtoyons les installations portuaires, le chemin de fer, le pont Victoria. Et l'on cherchera en vain dans le paysage - tous ces espaces industriels ou semiindustriels, usines électriques et pétrolières, espaces commerciaux, chantiers de construction (avec les grues dans le ciel gris, les pelles mécaniques qui creusent), tous ces terrains vagues ces lieux de vie que sont les quartiers. Le cinéaste nous livre quel- 
ques portraits de rue, clichés de passants qui semblent habiter l'endroit, mais c'est surtout au béton que nous sommes livrés.

Comment montrer, dans Le temps qu'il fait, le temps qu'il fait? Parce qu'il s'agit du temps de l'adaptation à la mondialisation, on filmera un paysage industriel: Ultramar, Shell, Petro Canada, Canada Parker, Maple Leaf. Les travailleurs parlent, ces travailleurs «libres et nus du capitalisme» (Deleuze et Guattari, 1980 , p. 532). On est devenu journalier, on raconte comment, et les travellings accusent. En effet, l'exaspération du cinéaste passe par une exaspération de la technique, qui est ici tout en travellings incessants et obsédants. Et c'est par là que tout ce visible de la ville se mue en visions de la ville. Ce sont deux statuts, écrit Comolli, qui peuvent co-exister: "La ville du cinéaste ne serait donc pas seulement le visible de la ville. Il se peut, même, que cet écart par rapport au visible soit la dimension juste du cinéma..." (1994, p. 17). Dans la perspective qui est mienne, ce que cette vision représente, c'est le commentaire visuel de l'auteur sur la parole recueillie, comme une conscience réfléchissante, un "cogito proprement cinématographique" (Pasolini, 1976, p. 18). Ce sont les propres affects de l'auteur qui transitent ici par une formalisation du paysage. Ces visions, mi-objectives, mi-subjectives, sont une réflexion de la parole dans un regard, une conscience-de-soi-caméra. Elles viennent réfléchir le contenu des paroles dans une "conscience-caméra devenue autonome" (Pasolini, 1976, p. 108).

Une séquence est exemplaire de la façon dont le cinéaste exprime son indignation: un train passe et le cinéaste a posé sa caméra tout près des rails, à ras du sol, si bien que pendant toute la durée - fort longue -- du passage du train, le spectateur ne voit rien d'autre qu'une tache noire mouvante. Le champ de la représentation est ici comme effacé par le processus cinématographique lui-même, comme si nous étions au-delà d'une possibilité de sémiotisation. Plan formellement exaspérant, qui ne fait que traduire visuellement (là se loge le commentaire) l'exaspération grandissante du cinéaste et de ses sujets. "On ne peut s'en remettre, écrit Guattari, à la pluie et au beau temps capitalistes..." (1986, p. 120). Il y a à cet égard, dans Le temps quil fait, un beau parallèle: jeu des doigts à la bourse et jeu des doigts d'un musi- 
cien oriental dans le métro de Montréal. Entre l'abstraction du capital et la singularité artiste, à quoi s'en remettre? Nous en sommes là, dans les paysages de Sylvain L'Espérance, qui ne soustendent plus un pays, mais une économie dévastatrice.

Ce que la caméra montrait dans le cycle abitibien de Pierre Perrault, c'est la désertification des lieux et les stigmates de la souffrance sur les visages. On retrouve cette même "physicité " des lieux chez L'Espérance (caméra topographique), mais aussi des visages, des mains, des silences (caméra prosopographique). Dans le cycle abitibien, Perrault filmait la ruine, le vide, la désertification: maisons abandonnées, granges déhanchées, terres en friche, taudis, clos écroulés, cimetières de voitures autour des solages, etc. Dans Les Printemps incertains et Le temps qu'il fait, si L'Espérance filme aussi la ruine et le vide, ce n'est toutefois plus le même désert, le même paysage déserté et désertique. C'est une autre esthétique des restes, restes urbains, c'est une autre forme de mélancolie liée au délabrement, mais c'est une même éthique cinématographique: celle de la constitution du regard et de la mémoire par le cinématographe, "ce réveil des traces dont le cinéma porte en lui l'urgence depuis son début même... (Comolli, 1994, p. 50). Comme s'il fallait faire le deuil du lieu, ne pas le perdre, le re-trouver.

Pierre Perrault faisait l'apologie d'une collectivité. La dimension à la fois épique et utopique de son cinéma a bel et bien disparu des documentaires de la nouvelle génération, mais dans lesquels une même démarche cinématographique est à l'œuvre, une démarche où rien n'est commenté par la voix off, mais où "tout est pénétré, regardé intensivement, et redonné à voir " (Daudelin, 2001, p. 50). Une démarche dans laquelle le cinéaste, avant de se faire voyant, se fait d'abord écoutant, et pour qui le profilmique (la parole), parce qu'elle est captée en direct et dans son lieu, acquiert une valeur inestimable.

Université de Montréal 


\section{NOTES}

1. L'expression est de Jean-Louis Comolli. Voir ses deux articles consacrés à l'esthétique du direct publiés dans Les Cahiers du cinéma en 1969 (les numéros 209 et 212).

2. On sait que c'est principalement en France, aux États-Unis et au Québec que cette évolution technique a pu se transformer en véritable révolution esthétique, à la fois du côté du documentaire comme de celui de la fiction.

3. Formulation qui renvoie au titre de l'article d'André Gaudreault et de Philippe Marion dans Cinémas: "Dieu est l'auteur des documentaires" (Gaudreault et Marion, 1994).

4. Spécifions une tradition, car tous les cinéastes ne s'engageront pas dans cette pratique, même si elle est dominante.

5. Voir Gaudreault et Marion (1994).

6. Dans le Petit Robert, à "expliquer".

\section{RÉFÉRENCES BIBLIOGRAPHIQUES}

Comolli, 1994 : Jean-Louis Comolli, "La ville filmée ", Regards sur la ville, Paris, Supplémentaires, centre Georges-Pompidou, 1994, p. 13-61.

Comolli, 1997 : Jean-Louis Comolli, "Le miroir à deux faces", Arrêt sur histoire, Paris, Supplémentaires, centre Georges-Pompidou, 1997, p. $11-45$.

Daudelin, 2001 : Robert Daudelin, "Van Der Keuken: L'œil au-dessus du puits ", 24 Images, $\mathrm{n}^{\circ} 106$, printemps 2001, p. 50.

Deleuze, 1985: Gilles Deleuze, Cinéma 2: Limage-temps, Paris, Minuit, 1985.

Deleuze et Guattari, 1980 : Gilles Deleuze et Félix Guattari, Capitalisme et schizophrénie. Mille Plateaux, Paris, Minuir, 1980.

Gaudreault et Marion, 1994 : André Gaudreault et Philippe Marion, "Dieu est l'auteur des documentaires ", CiNéMAS, vol. 4, n 2, hiver 1994, p. 11-26.

Gauthier, 1983: Guy Gauthier, "Une écriture du réel », dans Guy Gauthier (dir.), Écritures de Pierre Perrault, Montréal/Paris, Cinémathèque québécoise/Édilig, $n^{\circ} 11$, 1983 , p. 25 à 33.

Guattari, 1986: Félix Guattari, Les Années d'hiver (1980-1985), Paris, Bernard Barrault, 1986.

Pasolini, 1976: Pier Paolo Pasolini, L’Expérience hérétique, Paris, Payor, 1976.

Perrault, 1972 : Pierre Perrault, Un pays sans bon sens, Montréal, Éditions Lidec, 1972.

Perrault, 1983: Pierre Perrault, "Cinéma du réel et cinéma de fiction: vraie ou fausse distinction? Dialogue entre Pierre Perrault et René Allio ", dans Gauthier, 1983, p. 42 à 57.

Perrault, 1985: Pierre Perrault, De la parole aux actes, Montréal, L'Hexagone, 1985.

Perrault, 1995 : Pierre Perrault, L'Oumigmatique ou l'objectif documentaire. Essai, Montréal, l'Hexagone, 1995.

Rancière, 1997 : Jacques Rancière, "L'Inoubliable ”, dans Arrêt sur histoi ;, Paris, Supplémentaires, centre Georges-Pompidou, 1997, p. 47 à 70.

Thérien, 1999: Gilles Thérien, "Bête numineuse", dans Paul Warren (dir.), Pierre Perrault, cinéaste-poète, Montréal, l'Hexagone, 1999, p. 163 à $17^{\circ}$. 

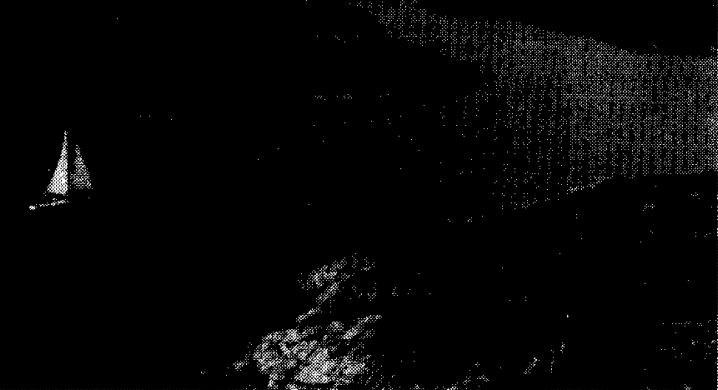

\section{Voiles bas et en travers (Pierre Perrault, 1983)}

À travers la trace physique, tout l'effort cinématographique est de faire lever, de soulever de terre ou de mer, une puissance métaphysique, un esprit des lieux.

Collection Cinémathèque québécoise

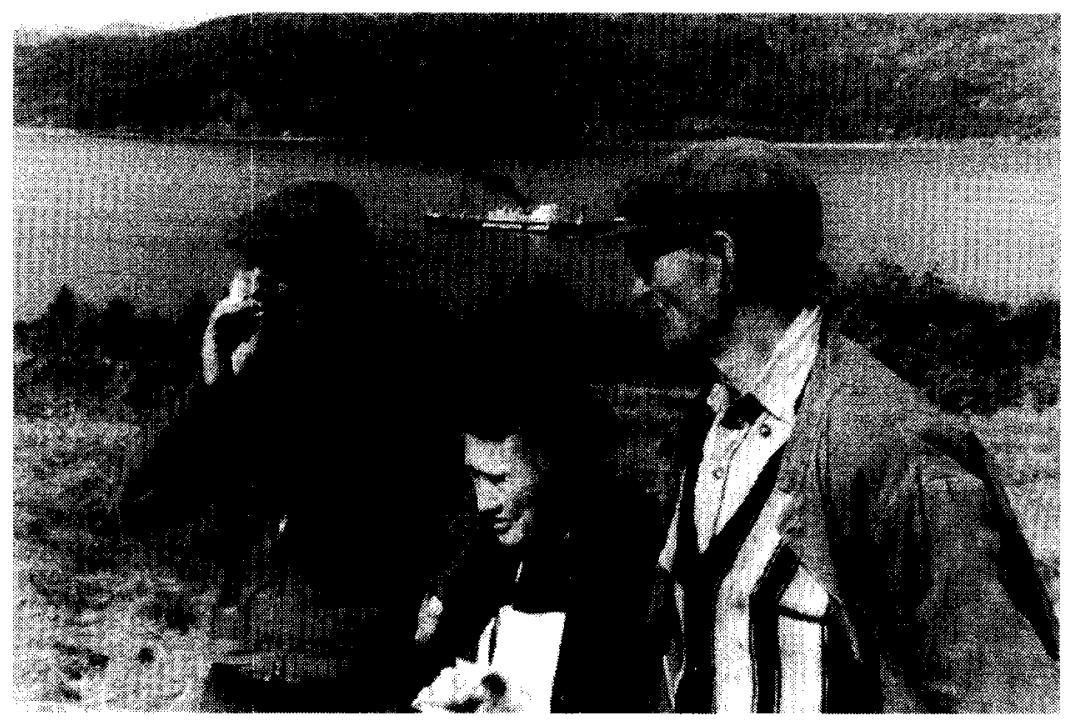

\section{Le Règne du jour (Pierre Perrault, 1966)}

Une parole lumineuse en accord avec son paysage.

Collection Cinémathèque québécoise 


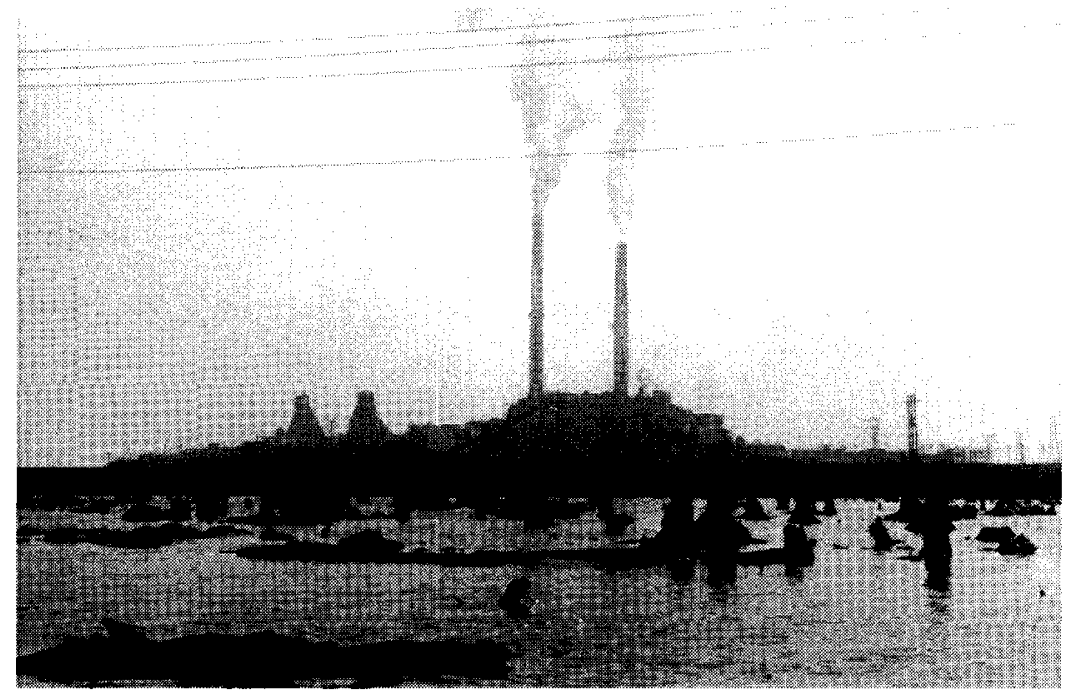

\section{Un royaume vous attend (Pierre Perrault et Bernard Gosselin, 1975)}

Solitude et résonnance de la parole dans un paysage qu'on se prépare à déserter.

Collection Cinémathèque québécoise 


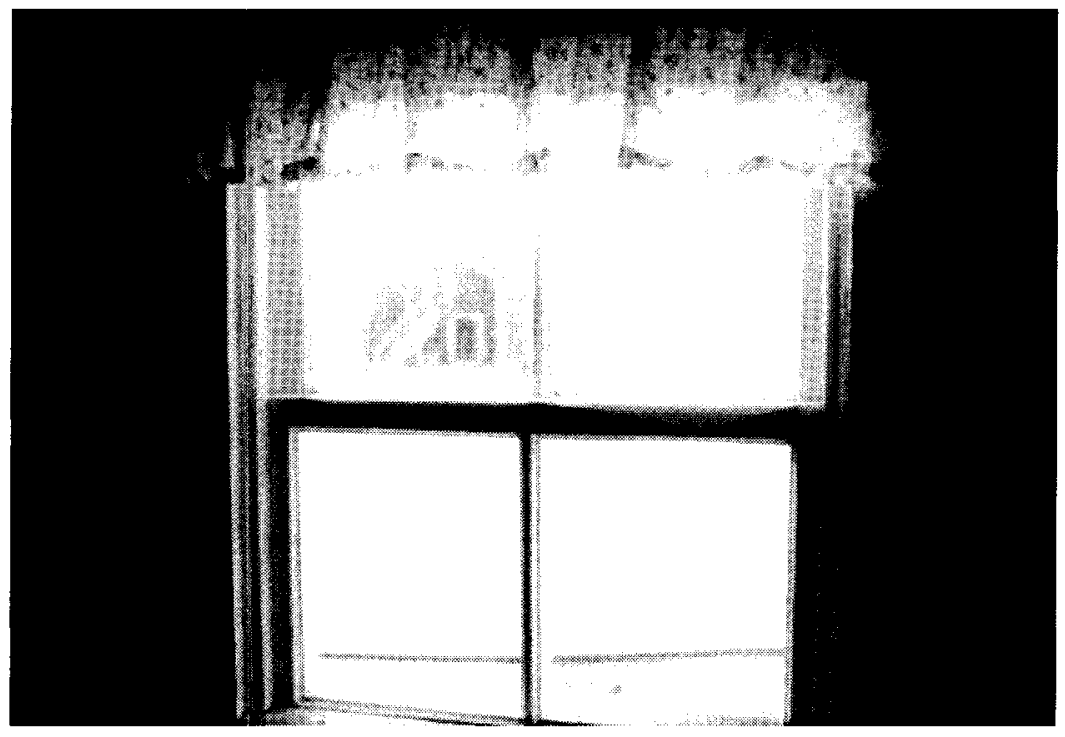

\section{Avant le jour (Lucie Lambert, 1999)}

Quand le regard-paysage vient au secours des mots pour faire entendre leur silence.

Photographie de plateau, collection personnelle

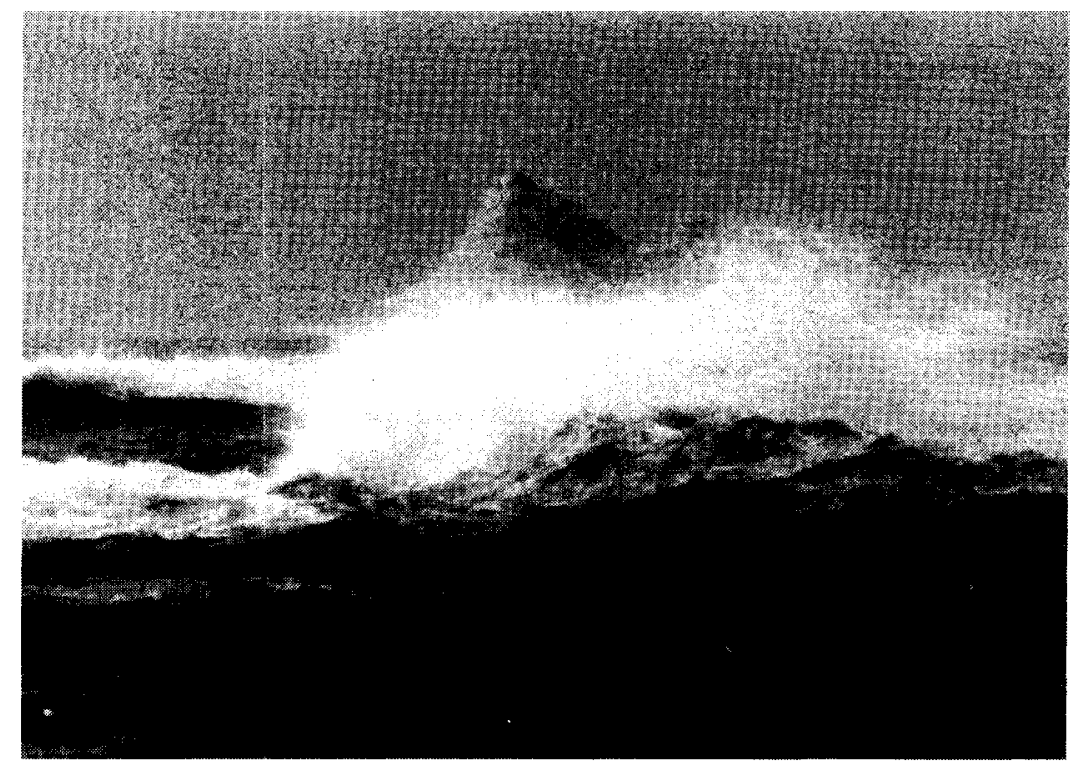

\section{Avant le jour (Lucie Lambert, 1999)}

La crispation formelle du paysage, s'irréalisant dans la blancheur de l'hiver, vient communier avec la douleur exprimée par les mots.

Photographie de plateau, collection personnelle 